\title{
A new improved protocol for in vitro intratubular dentinal bacterial contamination for antimicrobial endodontic tests: standardization and validation by confocal laser scanning microscopy
}

\author{
Flaviana Bombarda de ANDRADE'1, Marcela Paola Castro ARIAS'1, Amanda Garcia Alves MALIZA', Marco Antonio \\ Hungaro DUARTE1', Márcia Sirlene Zardin GRAEFF², Pablo Andrés AMOROSO-SILVA', Raquel Zanin MIDENA', \\ Ivaldo Gomes de MORAES ${ }^{1}$
}

\begin{abstract}
1- Department of Operative Dentistry, Endodontics and Dental Materials, Bauru School of Dentistry, University of São Paulo, Bauru, SP, Brazil 2- Integrated Research Center, Bauru School of Dentistry, University of São Paulo, Bauru, SP, Brazil.
\end{abstract}

Corresponding address: Flaviana Bombarda de Andrade - Faculdade de Odontologia de Bauru - Universidade de São Paulo - Al. Dr. Octávio Pinheiro Brisolla 9-75 - Vila Universitária - Bauru - SP - Brazil - 17012-901 - Phone: +55 (14) 32358344 / +55 (14) 997501313 - Fax: +55 (14) 32358323 - e-mail: flaviana@fob.usp.br

Submitted: July 7, 2014 - Modification: November 18, 2014 - Accepted: November 18, 2014

\section{ABSTRACT}

$\mathrm{O}$ bjectives: To compare three methods of intratubular contamination that simulate endodontic infections using confocal laser scanning microscopy (CLSM). Material and Methods: Two pre-existing models of dentinal contamination were used to induce intratubular infection (groups A and B). These methods were modified in an attempt to improve the model (group C). Among the modifications the following were included: specimen contamination for five days, ultrasonic bath with BHI broth after specimen sterilization, use of $\mathrm{E}$. faecalis during the exponential growth phase, greater concentration of inoculum, and two cycles of centrifugation on alternate days with changes of culture media. All specimens were longitudinally sectioned and stained with of LIVE/DEAD ${ }^{\circledR}$ for 20 min. Specimens were assessed using CLSM, which provided images of the depth of viable bacterial proliferation inside the dentinal tubules. Additionally, three examiners used scores to classify the CLSM images according to the following parameters: homogeneity, density, and depth of the bacterial contamination inside the dentinal tubules. Kruskal-Wallis and Dunn's tests were used to evaluate the live and dead cells rates, and the scores obtained. Results: The contamination scores revealed higher contamination levels in group $C$ when compared with groups $A$ and $B(p<0.05)$. No differences were observed between group $A$ and $B(p>0.05)$. The volume of live cells in group $C$ was higher than in groups $A$ and $B(p<0.05)$. Conclusion: The new protocol for intratubular infection resulted in high and uniform patterns of bacterial contamination and higher cell viability in all specimens when compared with the current methods.

Keywords: Confocal microscopy. Contamination. Dentin. Enterococcus faecalis.

\section{NTRODUCTI ON}

Bacteria and their toxins are the major cause of the development, progression, and persistence of pulp and periapical diseases ${ }^{12,17,25}$. Appropriate biomechanical preparations, assisted by irrigants and intracanal medications to promote the elimination of bacteria and their products, are required to obtain success in root canal treatment ${ }^{11,14,22,23,30}$. A reason for the persistence of endodontic infections is bacterial penetration inside the dentinal tubules, rendering its elimination difficult ${ }^{10,27,29}$.

In in vitro experimental models of endodontic infection, the homogeneous contamination of dentinal tubules is difficult to achieve. However, it is an essential factor for validating and comparing the results of antimicrobial irrigant and intracanal medication testing ${ }^{16,18,27}$. 
Some authors verified this bacterial penetration by means of microbiological culture ${ }^{3,10}$ and histological analysis with Brown \& Brenn staining ${ }^{15,16}$, both of them showing interesting results and images of dentinal contamination; however, they are time-consuming methods. Alternatively, scanning electron microscopy (SEM) was used to verify the disinfection of the root canal walls $s^{4,15,22,28}$. Because SEM achieves extremely high magnification, each field of analysis is therefore very small. Thus, many SEM images are necessary to accurately assess the outcome. Optical microscopy $(\mathrm{OM})^{16}$ and confocal laser scanning microscopy (CLSM) ${ }^{18,20,22,28}$ have lower magnification abilities than SEM, therefore OM and CLSM produce larger analysis fields, which means that less images are required.

Furthermore, with the help of fluorescent stains such as LIVE/DEAD ${ }^{\circledR}$ BacLight $^{T M}{ }^{20}$, it is possible to distinguish between live (stained with green) and dead (stained with red) bacteria by their color and to determine the viability profile and spatial distribution of bacteria inside the dentinal tubules.

Therefore, the aim of this study was to test and improve the recognized in vitro dentinal tubule contamination methods currently available in the literature ${ }^{3,10,16,18,20}$. Particularly, using CLSM and LIVE/DEAD ${ }^{\circledR}$ BacLight $^{T M}$ as evaluation tools, it aimed to develop a bovine root canal-based contamination protocol that produced a more homogeneous pattern of live bacterial distribution inside the dentine. It is expected that the use of this model will result in more accurate antimicrobial endodontic testing results.

\section{MATERI AL AND METHODS}

Fifteen bovine teeth were divided into three equal groups $(n=5)$ in order to test three distinct protocols of dentinal tubule contamination. The contamination was performed using the microorganism Enterococcus faecalis. The teeth of group A were contaminated according to the method of Haapasalo and Orstavik ${ }^{10}$ (1987), while group $\mathrm{B}$ followed the protocol of $\mathrm{Ma}$, et al. ${ }^{18}$ (2011), and group C consisted of a new method of contamination, described below. Three teeth were used as negative controls. These teeth were processed and evaluated in an identical fashion to the experimental groups, except that they did not undergo any contamination. Eight CLSM images were obtained for each tooth (four images of each half). Each experimental group was therefore comprised of forty images.

All specimens of the three groups were prepared using the following procedure. Freshly extracted bovine teeth were stored for 48 hours in $1 \%$ sodium hypochlorite solution for initial decontamination, and were subsequently immersed in distilled water.
The teeth crowns and five apical $\mathrm{mm}$ were removed. These radicular samples were standardized in 12 $\mathrm{mm}$ lengths using an Isomet saw (Isomet 1000, Buehler Ltd, Lake Bluff, IL, USA) with a diamond disk at $250 \mathrm{rpm}$, under irrigation. The root canals were prepared using $K$ files (Dentsply, York, PA, USA) up to \#120. Teeth with a wider root canal were discarded. The inorganic part of the smear layer was removed with $17 \%$ ethylenediaminetetraacetic acid (EDTA) (Biodinâmica Química e Farmacêutica, Ibiporã, PR, Brazil) for 5 min, and the canals were washed with deionized water. Two layers of red nail polish (L'Oréal Colorama, Rio de Janeiro, RJ, Brazil) were then used to cover the external surface of the samples. They were dried for $24 \mathrm{~h}$ before being autoclaved at $121^{\circ} \mathrm{C}$.

E. faecalis (ATCC 29212) was reactivated in Brain Heart Infusion broth (BHI, Difco, Kansas City, MO, USA) and maintained at $37^{\circ} \mathrm{C}$ for $24 \mathrm{~h}$. The bacterial culture was transferred to another $\mathrm{BHI}$ flask and incubated for further $24 \mathrm{~h}$ in order to achieve exponential growth. This culture was adjusted to McFarland standard No. $1\left(3 \times 10^{8} \mathrm{CFU} / \mathrm{mL}\right)$ using a spectrophotometer (Bel Photonics do Brasil Ltda, Osasco, SP, Brazil).

Group A contamination followed the protocol established by Haapasalo and Orstavik ${ }^{10}$ (1987). Briefly, following the preparation of cylindrical dentin specimens from bovine incisors, the tubules were opened by a four min treatment with $17 \%$ EDTA. The infection was then established over 21 days of incubation with E. faecalis, with daily culture media changes.

Group B contamination followed the protocol of Ma, et al. ${ }^{18}$ (2011). Briefly, following the preparation of cylindrical dentin specimens from bovine incisors, an E. faecalis suspension was added to a centrifuge tube containing the dentin specimen. The tubes were centrifuged in sequence at 1,400 , $2,000,3,600$, and $5,600 \mathrm{~g}$, over two cycles of 5 min for each speed. A fresh solution of bacteria was added between every centrifugation, and the solution that had penetrated through the dentin piece was discarded. Following centrifugation, all tubes were incubated at $37^{\circ} \mathrm{C}$ in $\mathrm{BHI}$ broth under aerobic conditions for $24 \mathrm{~h}$, in order to facilitate bacterial recovery.

The group $\mathrm{C}$ contamination model proceeded as follows (flowchart in Figure 1). First, $800 \mu \mathrm{L}$ of sterilized $\mathrm{BHI}$ was inserted in each microtube (Axygen Scientific, Union City, CA, USA) containing the dentine specimen. Activation was performed in an ultrasonic bath for 15 min to allow for the maximum penetration of the culture medium into the dentinal tubules prior to the bacterial contamination step. Contamination using the standardized inocula was performed over five days, with centrifugation on alternate days. Following 
adjustment of the E. faecalis culture to McFarland standard No. 1 , it was incubated again at $37^{\circ} \mathrm{C}$ for seven hours in order to achieve exponential growth. This amount of time $(7 \mathrm{~h})$ was defined in a pilot study (data not shown). This inoculum $(800 \mu \mathrm{L})$ was inserted into the microtubes with the samples and centrifuged (Eppendorf 5417R centrifuge, Eppendorf, Hamburg, Germany) as described above for group $B$. The inoculum was renewed at each cycle of centrifugation. Following the eight centrifugation cycles, sterilized BHI broth was inserted into the microtubes, agitated in a vortex (Vortex-mix VX200, Edison, NJ, USA), and incubated at $37^{\circ} \mathrm{C}$ for $24 \mathrm{~h}$.

Following incubation (day two), the samples were agitated in a vortex for $10 \mathrm{~s}$, and the inocula from the microtubes were discarded. One milliliter of sterilized $\mathrm{BHI}$ broth was inserted, followed by a centrifuge cycle of $3,600 \mathrm{~g}$ for $5 \mathrm{~min}$ at $25^{\circ} \mathrm{C}$, and the microtubes were incubated again at $37^{\circ} \mathrm{C}$ for $24 \mathrm{~h}$.

On the third day, a new inoculum of E. faecalis at exponential growth phase after seven hours of last subculture was inserted into the sample tubes, and the centrifugation protocol (twice at each speed, at $25^{\circ} \mathrm{C}$ ) was repeated. On the fourth day, the procedures were as described for the second day. On the fifth day, the samples were removed from the microtubes and observed with CLSM.

\section{FIRST DAY}
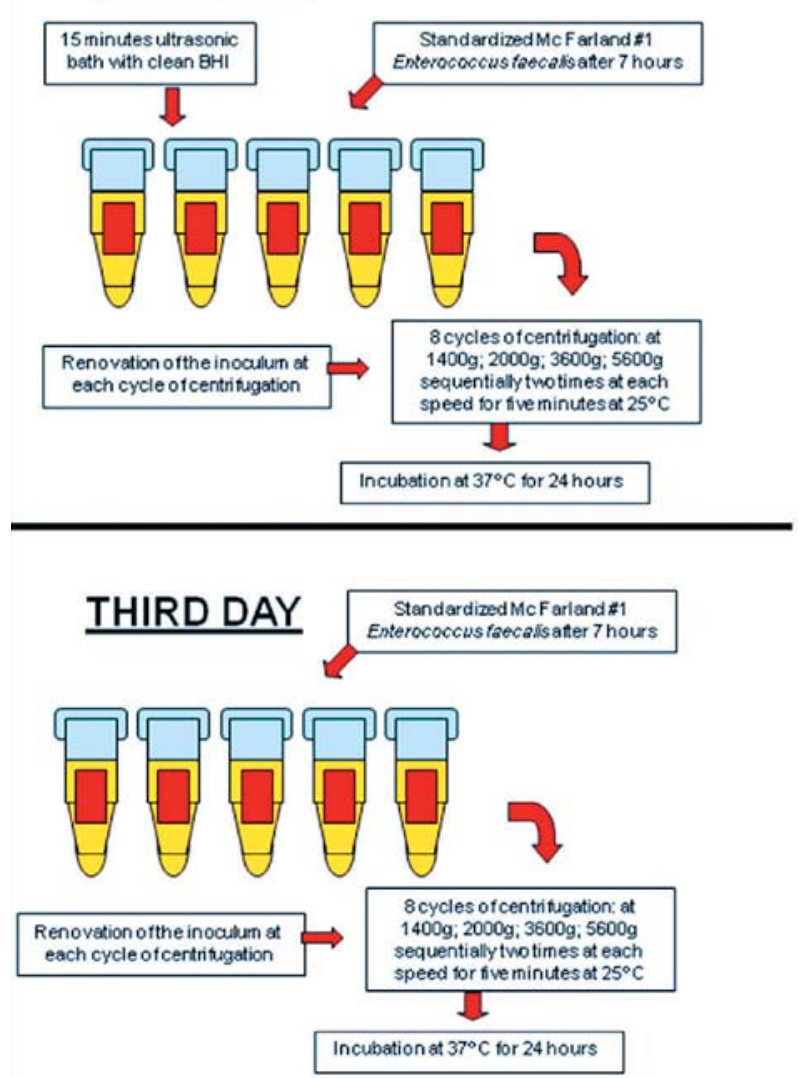

Figure 1- Flowchart of the group $\mathrm{C}$ contamination protocol
Thus, group A underwent three weeks of contamination, group B $24 \mathrm{~h}$, and group C five days. All three groups were analyzed by CLSM and fluorescent LIVE/DEAD ${ }^{\circledR}$ BacLight $^{\mathrm{TM}}$ Bacterial Viability stain (Molecular Probes, Eugene, OR, USA) to verify the bacterial penetration.

Following the contamination procedures, all specimens were longitudinally sectioned using an Isomet saw with a diamond disc. Constant irrigation with a sterilized saline solution occurred during the sectioning process. The smear layer produced by these cuttings was removed with $17 \%$ EDTA for $10 \mathrm{~min}$, and the samples were washed in sterilized saline solution. The halves of the dentinal tubes were stained with $30 \mu \mathrm{L}$ of LIVE/DEAD ${ }^{\circledR}$ stain for 20 min. This kit contains SYTO $9^{\circledR}$ dye, which stains live bacteria with a green pigment, and propidium iodine dye, which stains dead bacteria with a red pigment, thus enabling the easy identification of viable bacteria.

The samples were examined with an inverted Leica TCS-SPE confocal microscope (Leica Microsystems GmbH, Mannheim, Baden-Württemberg, Germany) using a 40X magnification oil lens. Eight sequential images were taken of each dentinal tube; four of the cervical third and four of the medial third. In total, 40 images were taken from each group. The images were obtained by using 23 sections of $1 \mu \mathrm{m}$ step size, in a format of $1024 \times 1024$ pixels. The images

\section{SECOND DAY}

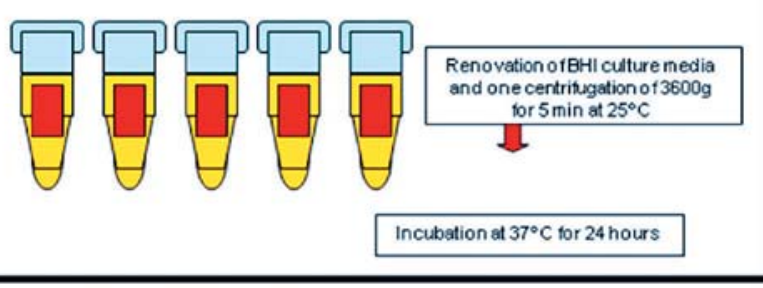

\section{FOURTH DAY}

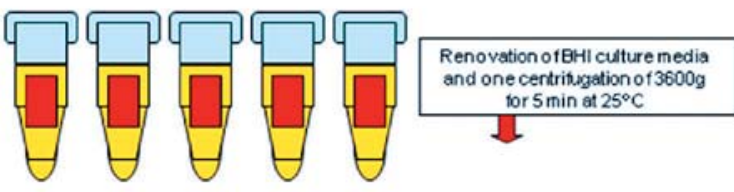

Incubation at $37^{\circ} \mathrm{C}$ for 24 hours

\section{FIFTH DAY}
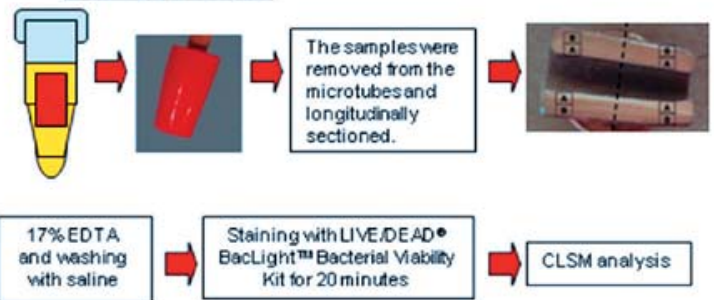
were acquired and fragmented using the Leica Application Suite-Advanced Fluorescence software (LAS AF, Leica, Mannheim, Baden-Württemberg, Germany).

For an objective analysis, the CLSM images were fragmented into many layers and were converted into "tiff" format by the LAS AF software. These images were exported to the bioImageL TM v21 software $^{7}$ in order to quantify the amounts of live (green) and dead (red) bacteria. The image layers were used to reconstruct the sections of the contaminated tubules in 3D form for an evaluation of the volume and distribution of the contamination. Each layer was evaluated individually with the "Surface and Volume Distribution" function. Bacterial viability and biovolume data were obtained from the analysis of each layer. These data were evaluated statistically with the Kruskal-Wallis and
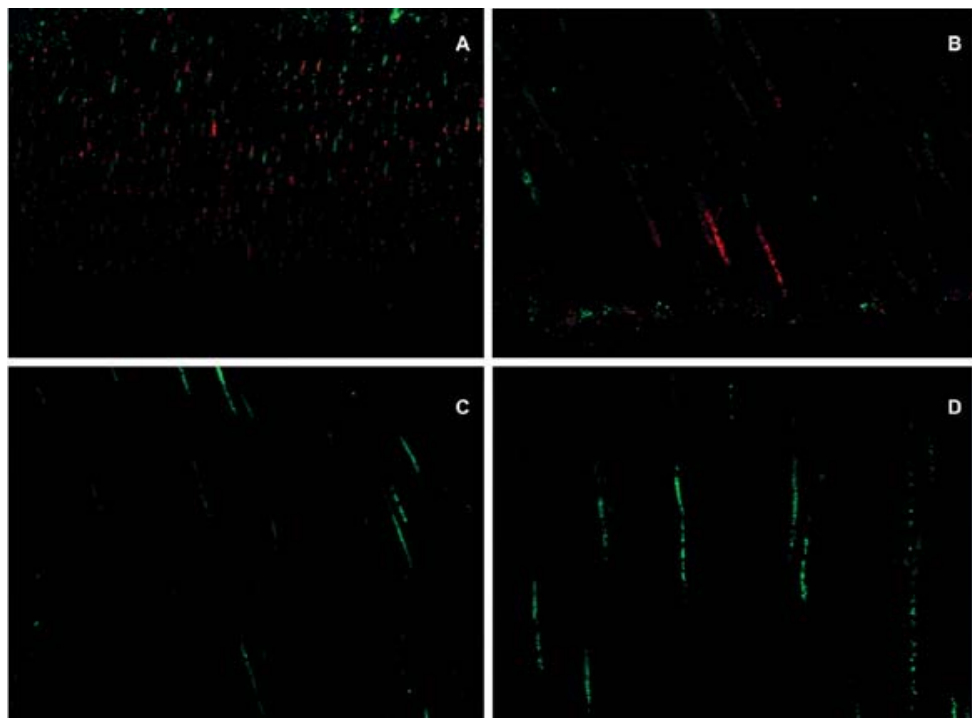

Figure 2- Confocal laser scanning microscopy (CLSM) images following contamination with E. faecalis. A- Longitudinal view of the medial third of a root canal following group A contamination. Low level penetration of bacteria inside the tubules can be observed. Red color denotes dead bacteria; B- Longitudinal view of the cervical third of a root canal following group $\mathrm{A}$ contamination. Compared to Figure $2 \mathrm{~A}$, there are slightly more bacteria inside the tubules and some of them are green, denoting live bacteria; C- Longitudinal view of the cervical third of a root canal following group B contamination. A small amount of live bacteria can be observed; $D$ - Longitudinal view of the medial third of the root canal following group $B$ contamination. A small amount of live bacteria can be observed
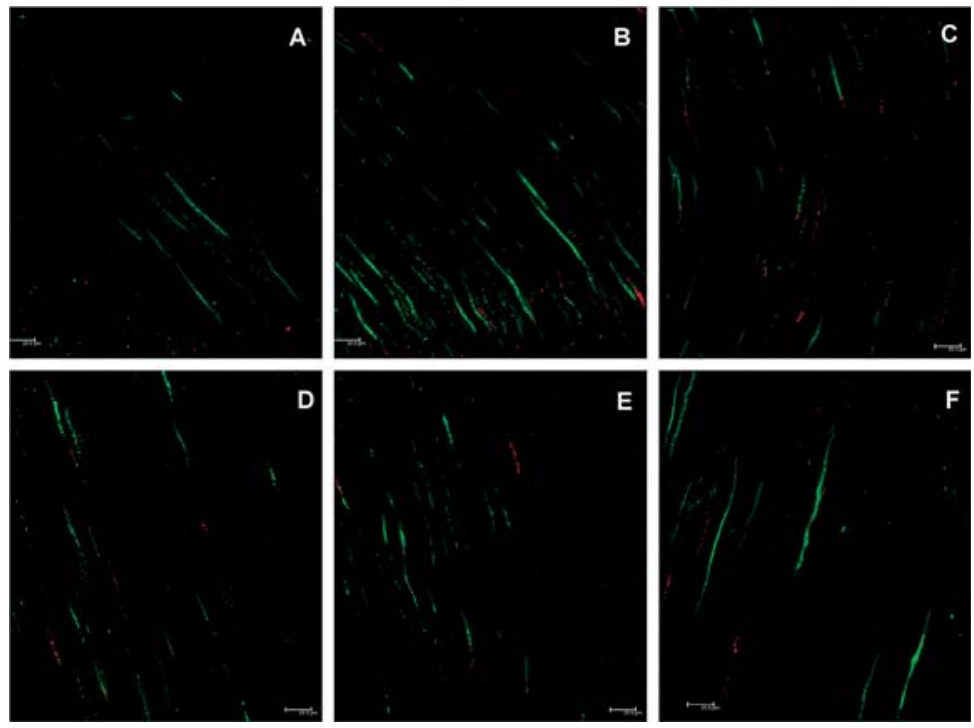

Figure 3- Confocal laser scanning microscopy (CLSM) images following group C contamination protocol. A, B, and CLongitudinal views of the cervical third of a root canal. A greater quantity of bacteria and a predominance of live (green) bacteria can be observed; D, E, and F- Longitudinal views of the medial third of a root canal. Live bacteria can be observed. The medial third, as in all figures, is less contaminated than the cervical third 
Dunn's tests. A p value of $<0.05$ was considered statistically significant.

In addition to the objective CLSM analysis, a subjective evaluation of contamination was performed by three blinded calibrated examiners using the CLSM images. Classification was defined according to the level of homogeneity, density, and depth of the bacterial contamination inside the dentinal tubules. Prior to the evaluation, the examiners were trained by examining other CLSM images of intradentinal contamination, as well as observing the images obtained from our three experimental groups, with no knowledge of which group the images were from.

The images were classified according to the following scores (determined in this study): 0 , no

\section{Intratubular Contamination by CLSM}

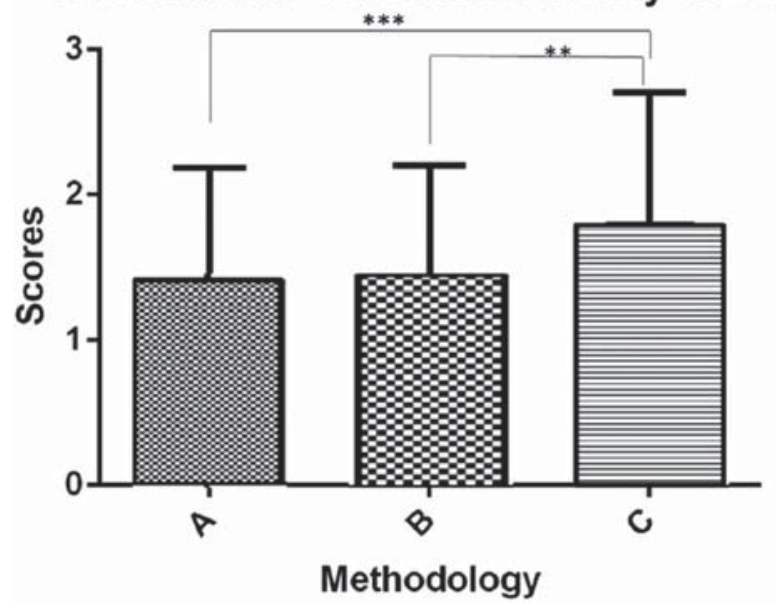

Figure 4- Classification of scores of intratubular contamination observed in the confocal laser scanning microscopy (CLSM) images, performed by three examiners $(A \times C, p=0.015)(B \times C, p=0.022)$. The symbol ** shows the statistical difference between groups $B$ and $C(p<0.05)$, and the symbol ${ }^{* *}$ shows the statistical difference between groups $A$ and $C(p<0.01)$

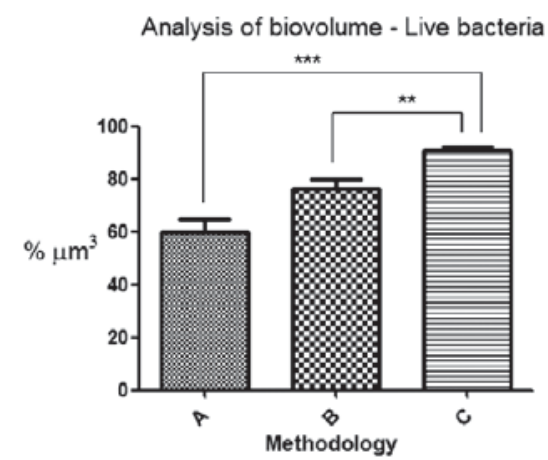

contamination; 1 , low contamination; 2, moderate contamination; and 3, high contamination. No contamination was defined as less than one-fourth of the analyzed field showing live bacteria inside the dentinal tubules. Low contamination was defined as half of the analyzed field showing live bacteria inside the tubules. Moderate contamination was defined as $3 / 4$ of the analyzed field showing live bacteria inside the tubules, and high contamination was defined as all of the analyzed field showing live bacteria, along with high bacterial proliferation observed within the dentinal tubules. The data were analyzed by the kappa agreement test, and a result of 0.7 (classified as good) was obtained. The subjective analysis was done by observation of the composite image from each site, i.e., each CLSM image was composed of 23 images at $1 \mu \mathrm{m}$ intervals, giving a final composite image of $23 \mu \mathrm{m}$ in depth. After the normality test, the KruskalWallis and Dunn's tests were used to perform the statistical analysis. $p$ values $<0.05$ were considered statistically significant.

\section{RESULTS}

The CLSM images obtained from group C demonstrated a greater bacterial penetration from the main root canal. This contamination was more dense and homogeneous, and a greater quantity of live bacteria were present (Figures 2 and 3).

After classifying the CLSM images in scores, the examiners observed greater ap<0.05); however, no differences were noted between groups $A$ and $B(p>0.05)$ (Figure 4).

The data obtained with the bioImageL v21 software, following the analysis and quantification of the biovolume of live and dead bacteria, produced the average values shown in Figure 5. KruskalWallis and Dunn's tests demonstrated a statistically significant difference between groups $\mathrm{A}$ and $\mathrm{C}$, and between groups $B$ and $C$ in the biovolumes of live

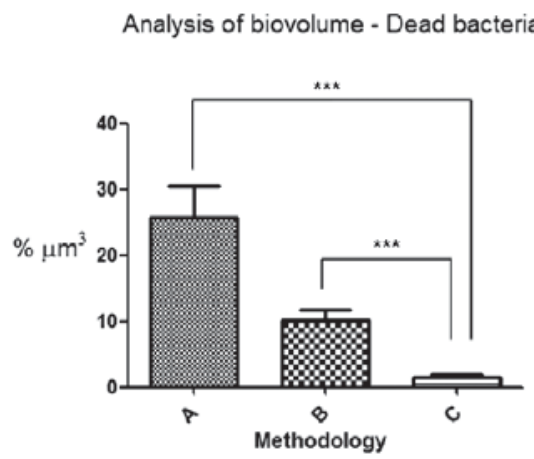

Figure 5- Quantification (in percentage of cubic micrometers) of the biovolume of live and dead bacteria by biolmageL v21 software, from confocal laser scanning microscopy (CLSM) images. The symbol ** shows the statistical difference between groups $B$ and $C$ in the analysis of biovolume of live bacteria $(p<0.05)$, and the symbol ${ }^{* * *}$ shows the statistical differences between groups $A$ and $C$ in the analysis of biovolume of live bacteria, and between groups $A$ and $C$, and $B$ and $C$, in the analysis of biovolume of dead bacteria $(p<0.01)$ 
and dead bacteria $(\mathrm{p}<0.05)$.

The negative controls did not show any bacteria at the images.

\section{DISCUSSION}

This study showed that a novel method for the intratubular contamination of bovine dentine is superior to previously reported contamination protocols ${ }^{10,18}$. This was demonstrated both by subjective score analyses and by quantitatively comparing the biovolume of live and dead bacteria inside the dentinal tubules.

Bovine teeth are commonly used as an experimental substitute for human teeth due to their ease of availability ${ }^{26}$. However, compared to human dentin, bovine dentin has a higher concentration of dentin tubules per square millimeter, although this difference is small. On average, the diameter of the dentin tubules of bovine teeth is greater than that of human teeth, although this difference is not statistically significant. The percentage of intertubular dentin in bovine teeth is the same as in humans ${ }^{24,26}$. In this study, the bacterial tubule penetration procedure was standardized, and since bovine tubules are generally larger, this process was easier to achieve than if human teeth had been used. A further advantage of using bovine teeth is that human teeth can show greater variations, due to age, microanatomy, and degree of tooth mineralization.

Haapasalo and Orstavik ${ }^{10}$ (1987) revolutionized the study of antimicrobial substances by introducing a new protocol for dentin contamination. This technique involved contamination with a pure colony of $\mathrm{E}$. faecalis for 21 days, followed by evaluation with SEM and Brown and Brenn staining. For medication tests, specimens infected for three weeks were used, and a layer of nail varnish was used to cover the root surfaces of the dentin blocks. This procedure rapidly became the method of choice for the evaluation of substances and treatment protocols in endodontics $8,13,19$.

Later, the same authors modified their protocol, using 14-day contamination periods and allowed cultures of dentin dust from sequential bur samples to be analyzed ${ }^{21}$. This modified protocol was effective for all microorganisms tested, with different times of contamination, and E. faecalis rapidly infected the whole length of the tubules. Furthermore, this protocol could be utilized in another study that evaluated the efficiency of nickel-titanium rotary instrumentation ${ }^{9}$. Additional improvements to the protocol by other scientists resulted in an even more rapid and simple methodology, requiring just seven days of contamination ${ }^{1,2}$.

In 2011, Ma, et al. ${ }^{18}$ introduced a new method that could induce biofilm on the dentin block surface beside the internal dentin contamination. The contamination was achieved in just $24 \mathrm{~h}$ and used a sequential centrifugation protocol; between every centrifugation, a fresh solution of bacteria was added. This protocol was developed to allow for the analysis of microorganisms in dentinal depth using fluorescence detected by CLSM. However, the experimental model comprised a small $4 \mathrm{~mm}$ dentin fragment to promote biofilm formation and contamination of the internal surface of the root canal, which have made the contamination easier.

This study confirmed that the classic protocol of Haapasalo and Orstavik ${ }^{10}$ (1987) (group A) produced a satisfactory amount of bacterial contamination, as confirmed by the biovolume and score analyses. However, the biovolume analysis also revealed large numbers of dead bacteria. Our results using the protocol of $\mathrm{Ma}$, et al. ${ }^{18}$ (2011) (group B) were deemed unsatisfactory, because the contamination included intermediate amounts of dead bacteria, and some areas revealed no contamination. Teeth from groups $B$ and $C$ received inocula between every centrifugation, but group $B$ had only one centrifugation and two centrifugations were performed in alternate days for group C. No statistical differences between the group $A$ and group B protocols were observed ( $p>0.05)$.

The new experimental model attempted to reproduce the oral environment of endodontic infections in bovine dentinal tubes $(12 \mathrm{~mm}$ length and canal internal diameter of $1.2 \mathrm{~mm}$ ), with an external surface covered with two layers of nail varnish. The goal was to ensure that the internal contamination occurred only via the root canal. This model, which is based on modifications to the group $B$ method, began by placing microcentrifuge tubes containing the samples and sterile culture media into an ultrasonic bath. This allowed for a greater penetration of the culture medium into the tubules, thus providing nutrients for the subsequent contamination step, which lasted four days. An exponential phase inoculum of high cell density was used for contamination and the centrifuge protocol of $\mathrm{Ma}$, et al. ${ }^{18}$ (2011) was followed. In this manner, the bacteria were already in an optimal proliferation phase before being launched toward the dentinal tubules by the centrifugal force. A period of seven hours, defined in a pilot study through the growth curve, was used for waiting for the exponential growth of the microorganism. After 24 hours, new sterilized media was inserted and a single centrifugation (3,600 g for 5 minutes) was performed in order to supply additional nutrients for bacterial proliferation. On days 3 and 4, respectively, a new inoculum was added (after $7 \mathrm{~h}$ ) and new media were inserted, thus repeating the procedures of days one and two. On the fifth day, the samples were sectioned and stained for 
CLSM analysis.

All of the modifications introduced into this protocol were added gradually over many pilot studies, in order to obtain the best contamination. For instance, slicing the dentinal tubes into two halves was found to be preferable to fracturing because some fractured fragments in the pilot studies did not provide satisfactory CLSM images. Furthermore, the use of a diamond disk for slicing was necessary to obtain a regular and plain surface, which improved the focus adjustment and view. After slicing, the smear layer was removed with $17 \%$ EDTA for 10 minutes, and the image of microorganisms within the tubules could then be captured.

EDTA has the capacity of killing microorganisms, but is a weak acid. With proper application time, EDTA could promote removal of smear layer without elimination of microorganisms, which is why only EDTA was used. In addition to their cleaning ability, chelators may detach biofilms adhering to the root canal walls ${ }^{21,30}$, but it has been demonstrated that its antiseptic capacity is relatively limited ${ }^{6,28,30}$. In the present work, EDTA was applied for a short time (10 minutes) for the removal of the smear layer only. Therefore, the contamination of the specimens was higher and deeper than in the tests that confirmed that EDTA presents antimicrobial capacity. A pilot study was performed in order to verify the influence of this superficial EDTA application and any influence of this weak acid over the bacterial viability was not observed.

It may be noted that images in the paper of $\mathrm{Ma}$, et al. ${ }^{18}$ (2011) demonstrate a greater contamination of tubules than our new protocol. However, this can be explained by differences in dentine fragment preparation. In the previous study, the tooth was cut into four pieces, without a cement layer, thus allowing for easier microbial penetration. In the present work, the dentine fragment was larger, and it contained cement and an external nail polish layer. Therefore, bacteria could access the dentinal mass only by the main root canal. Another factor is that in the $\mathrm{Ma}$, et al. ${ }^{18}$ (2011) images, it is possible to observe stained tubules because the dye has an affinity for dentine collagen ${ }^{5}$.

The adoption of the new endodontic protocol studies of antimicrobial substances should ensure a greater accuracy of results, particularly regarding the avoidance of false negatives due to failed contamination. It is important that this procedure be repeated with human teeth in order to verify that a satisfactory standard of contamination occurs in narrow and curved canals, and that an accurate replication of the clinical conditions of endodontic infections is achieved.

\section{CONCLUSI ON}

This research demonstrates a new protocol for the contamination of bovine dentinal tubules. It promotes greater and more homogeneous live bacterial proliferation compared to other methods. These aspects are essential for antimicrobial studies using in vitro models of endodontic infections, since they produce more accurate results and prevent false negatives.

\section{ACKNOWLEDGEMENTS}

This research was supported by the National Council for Scientific and Technological Development (CNPq) and São Paulo Research Foundation (FAPESP) (process no. 2010/20186-3). The authors would like to thank Dr Luis Chavez de Paz for the facilities for using the bioimageL software.

\section{REFERENCES}

1- Atila-Pektaş B, Yurdakul P, Gülmez D, Görduysus O. Antimicrobial effects of root canal medicaments against Enterococcus faecalis and Streptococcus mutans. Int Endod J. 2013;46(5):413-8.

2- Bago I, Plečko V, Gabrić Pandurić D, Schauperl Z, Baraba A, Anić I. Antimicrobial efficacy of a high-power diode laser, photoactivated disinfection, conventional and sonic activated irrigation during root canal treatment. Int Endod J. 2013;46(4):339-47.

3- Berber VB, Gomes BP, Sena NT, Vianna ME, Ferraz CC, Zaia AA, et al. Efficacy of various concentrations of $\mathrm{NaOCl}$ and instrumentation techniques in reducing Enterococcus faecalis within root canals and dentinal tubules. Int Endod J. 2006;39(1):10-7.

4- Bergmans L, Moisiadis P, Huybrechts B, Van Meerbeek B, Quirynen M, Lambrechts P. Effect of photo-activated disinfection on endodontic pathogens ex vivo. Int Endod J. 2008;41(3):227-39. 5- Bryce G, O'Donnell D, Ready D, Ng Y, Pratten J, Gulabivala K. Contemporary root canal irrigants are able to disrupt and eradicate single- and dual-species biofilms. J Endod. 2009;35(9):1243-8. 6- Byström A, Sundqvist G. The antibacterial action of sodium hypochlorite and EDTA in 60 cases of endodontic therapy. Int Endod J. 1985;18(1):35-40.

7- Chávez de Paz LE. Image analysis software based on color segmentation for characterization of viability and physiological activity of biofilms. Appl Environ Microbiol. 2009;75(6):1734-9. 8- Cheng X, Guan S, Lu H, Zhao C, Chen X, Li N, et al. Evaluation of the bactericidal effect of Nd:YAG, Er:YAG, Er,Cr:YSGG laser radiation, and antimicrobial photodynamic therapy (aPDT) in experimentally infected root canals. Lasers Surg Med. 2012;44(10):824-31.

9- Coldero LG, McHugh S, MacKenzie D, Saunders WP. Reduction in intracanal bacteria during root canal preparation with and without apical enlargement. Int Endod J. 2002;35(5):437-46.

10- Haapasalo M, Orstavik D. In vitro infection and disinfection of dentinal tubules. J Dent Res. 1987;66(8):1375-9.

11- Haapasalo M, Shen Y, Qian W, Gao Y. Irrigation in endodontics. Dent Clin North Am. 2010;54(2):291-312.

12- Kakehashi S, Stanley HR, Fitzgerald RJ. The effects of surgical exposures of dental pulps in germ-free and conventional laboratory rats. Oral Surg Oral Med Oral Pathol. 1965;20:340-9.

13- Kamberi B, Bajrami D, Stavileci M, Omeragiq S, Dragidella $F$, Koçani $F$. The antibacterial efficacy of biopure MTAD in root canal contaminated with Enterococcus faecalis. ISRN Dent. 2012;2012:390526. 
14- Kishen A, Sum CP, Mathew S, Lim CT. Influence of irrigation regimens on the adherence of Enterococcus faecalis to root canal dentin. J Endod. 2008;34(7):850-4.

15- Kitagawa R, Kitagawa H, Izutani N, Hirose N, Hayashi M, Imazato $\mathrm{S}$. Development of an antibacterial root canal filling system containing MDPB. J Dent Res. 2014;93(12):1277-82.

16- Love RM. Enterococcus faecalis - a mechanism for its role in endodontic failure. Int Endod J. 2001;34(5):399-405.

17- Love RM, Chandler NP, Jenkinson HF. Penetration of smeared or nonsmeared dentine by Streptococcus gordonii. Int Endod J. $1996 ; 29(1): 2-12$.

18- Ma J, Wang Z, Shen Y, Haapasalo M. A new noninvasive model to study the effectiveness of dentin disinfection by using confocal laser scanning microscopy. J Endod. 2011;37(10):1380-5.

19- Miranda RG, Santos EB, Souto RM, Gusman H, Colombo AP. Ex vivo antimicrobial efficacy of the EndoVac system plus photodynamic therapy associated with calcium hydroxide against intracanal Enterococcus faecalis. Int Endod J. 2013;46(6):499505.

20- Ordinola Zapata R, Bramante CM, Moraes IG, Bernardineli N, Gasparoto TH, Graeff MS, et al. Confocal laser scanning microscopy is appropriate to detect viability of Enterococcus faecalis in infected dentin. J Endod. 2008;34(10):1198-201.

21- Orstavik D, Haapasalo M. Disinfection by endodontic irrigants and dressings of experimentally infected dentinal tubules. Endod Dent Traumatol. 1990;6(4):142-9.
22- Ozdemir HO, Buzoglu HD, Calt S, Stabholz A, Steinberg D. Effect of ethylenediaminetetraacetic acid and sodium hypochlorite irrigation on Enterococcus faecalis biofilm colonization in young and old human root canal dentin: in vitro study. J Endod. 2010;36(5):842-6.

23- Paquette L, Legner M, Fillery ED, Friedman S. Antibacterial efficacy of chlorhexidine gluconate intracanal medication in vivo. J Endod. 2007;33(7):788-95.

24- Schilke R, Lisson JA, Bauss O, Geurtsen W. Comparison of the number and diameter of dentinal tubules in human and bovine dentine by scanning electron microscopic investigation. Arch Oral Biol. 2000;45(5):355-61.

25- Sundqvist G. Taxonomy, ecology, and pathogenicity of the root canal flora. Oral Surg Oral Med Oral Pathol. 1994;78(4):522-30. 26- Tanaka JL, Medici Filho E, Salgado JA, Sangado MA, Moraes LC, Moraes ME, et al. Comparative analysis of human and bovine teeth: radiographic density. Braz Oral Res. 2008;22(4):346-51. 27- Vera J, Siqueira JF Jr, Ricucci D, Loghin S, Fernández N, Flores $B$, et al. One-versus two-visit endodontic treatment of teeth with apical periodontitis: a histobacteriologic study. J Endod. 2012;38(8):1040-52.

28- Wong DT, Cheung GS. Extension of bactericidal effect of sodium hypochlorite into dentinal tubules. J Endod. 2014;40(6):825-9. 29- Wu MK, Dummer PMH, Wesselink PR. Consequences of and strategies to deal with residual post-treatment root canal infection. Int Endod J. 2006;39(5):343-56.

30- Zehnder M. Root canal irrigants. J Endod. 2006;32(5):389-98. 